\title{
GROUPS WITH THE SAME ORDERS OF SYLOW NORMALIZERS AS THE MATHIEU GROUPS
}

\author{
BEHROOZ KHOSRAVI AND BEHNAM KHOSRAVI
}

Received 17 October 2004

There exist many characterizations for the sporadic simple groups. In this paper we give two new characterizations for the Mathieu sporadic groups. Let $M$ be a Mathieu group and let $p$ be the greatest prime divisor of $|M|$. In this paper, we prove that $M$ is uniquely determined by $|M|$ and $\left|N_{M}(P)\right|$, where $P \in \operatorname{Syl}_{p}(M)$. Also we prove that if $G$ is a finite group, then $G \cong M$ if and only if for every prime $q,\left|N_{M}(Q)\right|=\left|N_{G}\left(Q^{\prime}\right)\right|$, where $Q \in$ $\operatorname{Syl}_{q}(M)$ and $Q^{\prime} \in \operatorname{Syl}_{q}(G)$.

\section{Introduction}

Let $G$ be a finite group. We denote by $\pi(G)$ the set of all prime divisors of $|G|$. It was proved that if $G$ is an alternating group, a finite projective special linear group, a Janko sporadic simple group, or a finite projective special symplectic group, then $G$ is characterizable by the orders of normalizers of its Sylow subgroups $[1,2,3,4,10]$.

Mazurov and Shi $[11,12,13,14]$ and Deng [7] proved that some of the almost sporadic simple groups are characterizable by the set of element orders. Chen [5] and A. Khosravi [9] proved that some of the almost sporadic simple groups are characterizable by the set of order components.

Notation 1. For a prime number $q$, we define

$$
n_{q}(G)=\left|N_{G}(Q)\right| \quad \text { where } Q \in \operatorname{Syl}_{q}(G) .
$$

In this paper, as the main result, the following theorems are proved.

Theorem 1.1. Let $M$ be a Mathieu group and let $G$ be a finite group. If for every prime number $q, n_{q}(M)=n_{q}(G)$, then $G \cong M$.

Theorem 1.2. Let $M$ be a Mathieu group and let $G$ be a finite group. Let $p$ be the greatest prime divisor of $|M|$. If $|G|=|M|$ and $n_{p}(M)=n_{p}(G)$, then $G \cong M$.

In this paper, all groups are finite. All further unexplained notations are standard and refer to [6], for example. Let $m$ be a positive integer and let $q$ be a prime number. Then $m_{q}$ denotes the $q$-part of $m$. In other words, $m_{q}=q^{k}$ if $q^{k} \| m$ (i.e., $q^{k} \mid m$ but $q^{k+1} \nmid m$ ). 
We would like to note that $\left|\mathbb{Z}_{6}\right|=\left|S_{3}\right|=6, \mathbb{Z}_{3} \triangleleft \mathbb{Z}_{6}$, and $\mathbb{Z}_{3} \triangleleft S_{3}$. Therefore $\left|N_{\mathbb{Z}_{6}}(P)\right|=$ $\left|N_{S_{3}}(P)\right|=6$, where $P \cong \mathbb{Z}_{3}$ is the 3 -Sylow subgroup of $\mathbb{Z}_{6}$ and $S_{3}$. But $\mathbb{Z}_{6} \not \equiv S_{3}$ and hence they are not characterizable by these conditions.

\section{Preliminary results}

The following lemma is an immediate consequence of [15, Theorem 2.1.17].

Lemma 2.1. Let $G$ be a $q$-group and let $|G|=q^{k}$, for some $k>0$. Then $p$ divides $\prod_{i=1}^{k}\left(q^{i}-\right.$ $1)$, for any prime divisor $p$ of $|\operatorname{Aut}(G)|$ with $p \neq q$.

LeMma 2.2 [8]. The following results hold:

(i) $\left|M_{11}\right|=7920, n_{2}\left(M_{11}\right)=16, n_{3}\left(M_{11}\right)=144, n_{5}\left(M_{11}\right)=20$, and $n_{11}\left(M_{11}\right)=55$;

(ii) $\left|M_{12}\right|=95040, n_{2}\left(M_{12}\right)=64, n_{3}\left(M_{12}\right)=108, n_{5}\left(M_{12}\right)=40$, and $n_{11}\left(M_{12}\right)=55$;

(iii) $\left|M_{22}\right|=443520, n_{2}\left(M_{22}\right)=128, n_{3}\left(M_{22}\right)=72, n_{5}\left(M_{22}\right)=20, n_{7}\left(M_{22}\right)=21$, and $n_{11}\left(M_{22}\right)=55$;

(iv) $\left|M_{23}\right|=10200960, n_{2}\left(M_{23}\right)=128, n_{3}\left(M_{23}\right)=144, n_{5}\left(M_{23}\right)=60, n_{7}\left(M_{23}\right)=42$, $n_{11}\left(M_{23}\right)=55$, and $n_{23}\left(M_{23}\right)=253$;

(v) $\left|M_{24}\right|=244823040, n_{2}\left(M_{24}\right)=1024, n_{3}\left(M_{24}\right)=216, n_{5}\left(M_{24}\right)=240, n_{7}\left(M_{24}\right)=$ $126, n_{11}\left(M_{24}\right)=110$, and $n_{23}\left(M_{24}\right)=253$.

Remark 2.3. Note that we can calculate the crucial normalizer orders $n_{11}\left(M_{11}\right), n_{11}\left(M_{12}\right)$, $n_{11}\left(M_{22}\right), n_{23}\left(M_{23}\right)$, and $n_{23}\left(M_{24}\right)$ without using GAP. For example, from the character table in atlas [6], we can see that there are two conjugacy classes of elements of order 11 in $M_{11}$, each of order 720, for a total of 1440 elements of order 11. Since a Sylow 11subgroup of $M_{11}$ has order 11, that means there are 144 Sylow 11-subgroups. Therefore, $n_{11}\left(M_{11}\right)=\left|M_{11}\right| / 144=55$. Similar calculations work in the other cases.

Lemma 2.4. Let $M$ be a Mathieu group and let $G$ be a finite group. If for every prime number $q, n_{q}(M)=n_{q}(G)$, then $|G|=|M|$.

Proof. Obviously if $Q$ is a $q$-Sylow subgroup of $G$, then $Q \leq N_{G}(Q)$ and hence $|Q|$ divides $\left|N_{G}(Q)\right|$. Also $|G|_{q}=|Q|$. Since $n_{q}(G)=n_{q}(M)$, we conclude that $\left(n_{q}(G)\right)_{q}=\left(n_{q}(M)\right)_{q}$. Therefore for every prime $q$, we have $|G|_{q}=|M|_{q}$. Hence $|G|=|M|$.

Remark 2.5. Lemma 2.4 shows that if Theorem 1.2 is proved, then Theorem 1.1 is an immediate consequence of Theorem 1.2. Therefore in the next section we only prove Theorem 1.2.

\section{Characterizations of the Mathieu groups}

We note that in the proof of Theorem 1.2, we use the classification of finite simple groups. We prove this theorem by using the following lemmas.

Lemma 3.1. Let $G$ be a finite group and let $M$ be $M_{11}, M_{12}$, or $M_{22}$. If $|G|=|M|$ and $n_{11}(M)=n_{11}(G)$, then $G$ has a normal series $1 \unlhd H \unlhd K \unlhd G$ such that $K / H$ is a simple group, 11 is a divisor of $|K / H|$, and $|G / K| \mid 5$.

Proof. Let $1=G_{0} \leq G_{1} \leq G_{2} \leq \cdots \leq G_{k}=G$ be a chief series of $G$. Since $11 \||M|$, there exists some $i$ such that 11 is a divisor of $\left|G_{i+1} / G_{i}\right|$. So let $G_{i}=H$ and $G_{i+1}=K$. Since $K / H$ 
is a chief factor of $G$, it follows that $K / H$ is a minimal normal subgroup of $G / H$. Also $K / H$ is characteristically simple which implies that $K / H$ be a simple group or a product of isomorphic simple subgroups. We know that 11||$K / H \mid$, but $11^{2} \nmid|G / H|$ and hence $K / H$ is a simple group.

Therefore $1 \unlhd H \unlhd K \unlhd G$ is a normal series of $G$, such that $K / H$ is a simple group and 11 is a divisor of $|K / H|$. Let $P$ be a 11-Sylow subgroup of $G$. Therefore $P \leq K$, which implies that $G=K N_{G}(P)$, by the Frattini argument. Then

$$
\frac{G}{K} \cong \frac{K N_{G}(P)}{K} \cong \frac{N_{G}(P)}{N_{G}(P) \cap K} \cong \frac{N_{G}(P)}{N_{K}(P)}
$$

Therefore $|G / K|$ is a divisor of $\left|N_{G}(P)\right|=55$. Since 11 divides $|K / H|$, we conclude that $|G / K|$ divides 5 and the proof is complete.

Lemma 3.2. Let $G$ be a finite group and let $M$ be $M_{11}, M_{12}$, or $M_{22}$. If $|G|=|M|$ and $n_{11}(M)=n_{11}(G)$, then $G \cong M$.

Proof. By Lemma 3.1, $G$ has a normal series $1 \unlhd H \unlhd K \unlhd G$ such that $K / H$ is a simple group, 11 is a divisor of $|K / H|$, and $|G / K|$ divides 5 . We claim that $|H|$ is a divisor of 5 . Since 11 divides $|K / H|$, we have $11 \nmid|H|$. If $q \in \pi(H)$, then let $Q$ be a $q$-Sylow subgroup of $H$. Now $H \triangleleft K$ and $Q \in \operatorname{Syl}_{q}(H)$. Hence by using the Frattini argument, we conclude that $K=H N_{K}(Q)$. We know that $C_{K}(Q) \unlhd N_{K}(Q)$ and $N_{H}(Q) \unlhd N_{K}(Q)$. Therefore $N_{H}(Q) \unlhd$ $C_{K}(Q) N_{H}(Q) \unlhd N_{K}(Q)$. Also

$$
\frac{K}{H} \cong \frac{H N_{K}(Q)}{H} \cong \frac{N_{K}(Q)}{H \cap N_{K}(Q)} \cong \frac{N_{K}(Q)}{N_{H}(Q)}
$$

and $K / H$ is a simple group. So $C_{K}(Q) N_{H}(Q)=N_{H}(Q)$ or $C_{K}(Q) N_{H}(Q)=N_{K}(Q)$. Now we consider these cases separately.

Case 1. If $C_{K}(Q) N_{H}(Q)=N_{H}(Q)$ and $|Q|=q^{k}$, then $C_{K}(Q)$ is a subgroup of $N_{H}(Q)$. Therefore $\left|C_{K}(Q)\right|$ divides $\left|N_{H}(Q)\right|$, and hence $\left|N_{K}(Q) / N_{H}(Q)\right|$ is a divisor of $\mid N_{K}(Q) /$ $C_{K}(Q) \mid$. We know that $N_{K}(Q) / C_{K}(Q)$ is isomorphic to a subgroup of $\operatorname{Aut}(Q)$. Also $11 \mid$ $|K / H|, 11 \nmid|H|, q \in \pi(H)$, and $|Q|=q^{k}$ (where $Q \in \operatorname{Syl}_{q}(H)$ ) which implies that $11 \mid$ $\prod_{i=1}^{k}\left(q^{i}-1\right)$, by Lemma 2.1 .

But easy calculations show that $11 \nmid \prod_{i=1}^{7}\left(2^{i}-1\right), 11 \nmid \prod_{i=1}^{3}\left(3^{i}-1\right), 11 \nmid(5-1)$, and $11 \nmid(7-1)$. Therefore this case is impossible.

Case 2. If $C_{K}(Q) N_{H}(Q)=N_{K}(Q)$, then

$$
\frac{K}{H} \cong \frac{N_{K}(Q)}{N_{H}(Q)} \cong \frac{C_{K}(Q) N_{H}(Q)}{N_{H}(Q)} \cong \frac{C_{K}(Q)}{C_{K}(Q) \bigcap N_{H}(Q)}
$$

Since 11 divides $|K / H|$, we conclude that 11 divides $\left|C_{K}(Q)\right|$, and hence $P \leq C_{K}(Q)$, where $P \in \operatorname{Syl}_{11}(G)$. Therefore $Q \leq C_{K}(P) \leq N_{G}(P)$. Hence $|Q|$ is a divisor of $\left|N_{G}(P)\right|=$ 55. Since $11 \nmid|Q|$, we conclude that $|Q|$ is a divisor of 5 , and hence $|H| \mid 5$. 
Therefore $|G / K| \cdot|H|$ is a divisor of 5. If $|G / K| \cdot|H|=5$, then $K / H$ is a simple group of order $|M| / 5$, which is a contradiction by the classification of finite simple groups (see [6, pages 239-241]). (There exists no simple group of order 1584, 19008, or 88704.) So $|G / K|=1,|H|=1$, which implies that $G$ be a simple group of order $M$. Since there exists only one simple group of order $|M|$, it follows that $G \cong M$.

Lemma 3.3. Let $G$ be a finite group and let $M$ be $M_{23}$ or $M_{24}$. If $|G|=|M|$ and $n_{23}(M)=$ $n_{23}(G)$, then $G \cong M$.

Proof. Similar to Lemma 3.1 we conclude that $G$ has a normal series $1 \unlhd H \unlhd K \unlhd G$ such that $K / H$ is a simple group, 23 is a divisor of $|K / H|$, and $|G / K|$ is a divisor of 11 . Since 23||$K / H \mid$ and $23^{2} \nmid|G|$, it follows that $23 \nmid|H|$. Also $23 \nmid \prod_{i=1}^{10}\left(2^{i}-1\right), 23 \nmid \prod_{i=1}^{3}\left(3^{i}-1\right)$, $23 \nmid(5-1), 23 \nmid(7-1)$, and $23 \nmid(11-1)$. Therefore if $Q$ is a $q$-Sylow subgroup of $H$, then $|Q|$ divides $n_{23}(G)=253$. Hence $|H|$ divides 11 . Therefore $|G / K| \cdot|H|$ is a divisor of 11 . If $|G / K| \cdot|H|=11$, then $K / H$ is a simple group of order $|M| / 11$, which is a contradiction by the classification of finite simple groups (see [6, pages 239-241]). (There exists no simple group of order 927360 or 22256640 .) So $|G / K|=1,|H|=1$, which implies that $G$ be a simple group of order $M$. Since there exists only one simple group of order $|M|$, it follows that $G \cong M$.

\section{Acknowledgments}

The authors express their gratitude to Professor Bell and the referee for useful suggestions which improved the manuscript. The first author would like to thank the Institute for Studies in Theoretical Physics and Mathematics (IPM) for the financial support. Also we want to thank Professor Thomas Breuer for his help in computing the orders of normalizers of Sylow subgroups of the Mathieu sporadic simple groups. We dedicate this paper to our parents: Professor Amir Khosravi and Mrs. Soraya Khosravi for their unending love. The first author was supported in part by a Grant from IPM (no. 82200031). We also have to thank the authors of the atlas [6] for the rich mine of information they have provided.

\section{References}

[1] J. Bi, A characterization of $L_{2}(q)$, J. Liaoning Univ. Nat. Sci. 19 (1992), no. 2, 1-4 (Chinese).

[2] A characterization of $L_{n}(q)$ by the normalizers' orders of their Sylow subgroups, Acta Math. Sinica (N.S.) 11 (1995), no. 3, 300-306.

[3] Characterization of alternating groups by orders of normalizers of Sylow subgroups, Algebra Colloq. 8 (2001), no. 3, 249-256.

[4] On the group with the same orders of Sylow normalizers as the finite simple group $S_{4}(q)$, Algebras Groups Geom. 18 (2001), no. 3, 349-355.

[5] G. Chen, A new characterization of sporadic simple groups, Algebra Colloq. 3 (1996), no. 1, 4958.

[6] J. H. Conway, R. T. Curtis, S. P. Norton, R. A. Parker, and R. A. Wilson, Atlas of Finite Groups, Oxford University Press, Eynsham, 1985.

[7] H. Deng, The number of isomorphism classes of finite groups with the set of element orders of almost sporadic simple groups, Southeast Asian Bull. Math. 22 (1998), no. 3, 265-271. 
[8] [GAP 00] The GAP Group, GAP—Groups, Algorithms, Programming-a System for Computational Discrete Algebra, Version 4.2; Aachen, St Andrews, 1999, http://wwwgap.dcs.st-and.ac.uk/ gap.

[9] A. Khosravi and B. Khosravi, A new characterization of almost sporadic groups, J. Algebra Appl. 1 (2002), no. 3, 267-279.

[10] B. Khosravi and B. Khosravi, Groups with the same orders of Sylow normalizers as the Janko groups, J. Appl. Algebra Discrete Struct. 3 (2005), no. 1, 23-31.

[11] V. D. Mazurov and W.-J. Shi, A note to the characterization of sporadic simple groups, Algebra Colloq. 5 (1998), no. 3, 285-288.

[12] W.-J. Shi, A new characterization of the sporadic simple groups, Group Theory (Singapore, 1987), de Gruyter, Berlin, 1989, pp. 531-540.

[13] _ Pure quantitative characterization of finite simple groups. I, Progr. Natur. Sci. (English Ed.) 4 (1994), no. 3, 316-326.

[14] The characterization of the sporadic simple groups by their element orders, Algebra Colloq. 1 (1994), no. 2, 159-166.

[15] M. Suzuki, Group Theory. I, Grundlehren der Mathematischen Wissenschaften, vol. 247, Springer, New York, 1982.

Behrooz Khosravi: Department of Pure Mathematics, Faculty of Mathematics and Computer Science, Amirkabir University of Technology (Tehran Polytechnic), 424 Hafez Avenue, Tehran 15914, Iran; Institute for Studies in Theoretical Physics and Mathematics (IPM), P.O. Box 19395-5746, Tehran, Iran

E-mail address: bkhosravi@aut.ac.ir

Behnam Khosravi: Department of Mathematics, Faculty of Mathematical Sciences, Shahid Beheshti University, Evin, Tehran 19838, Iran

E-mail address: khosravi@saba.tmu.ac.ir 


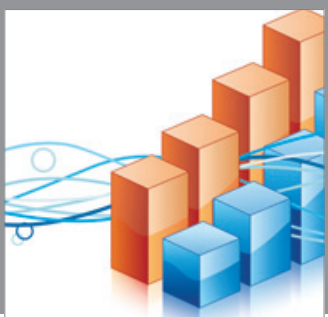

Advances in

Operations Research

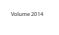

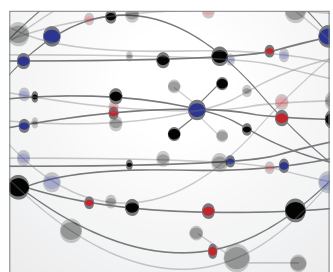

\section{The Scientific} World Journal
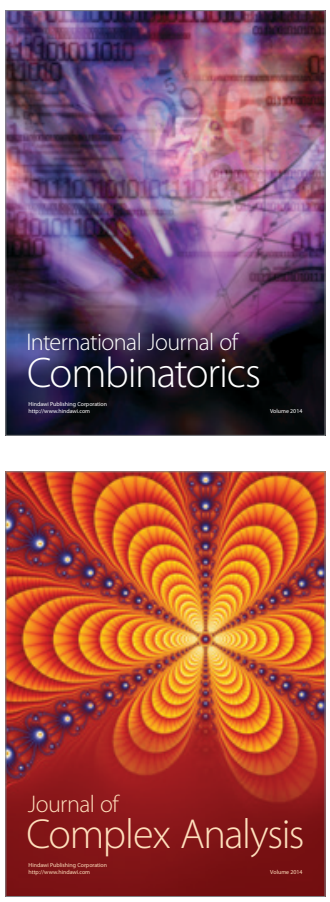

International Journal of

Mathematics and

Mathematical

Sciences
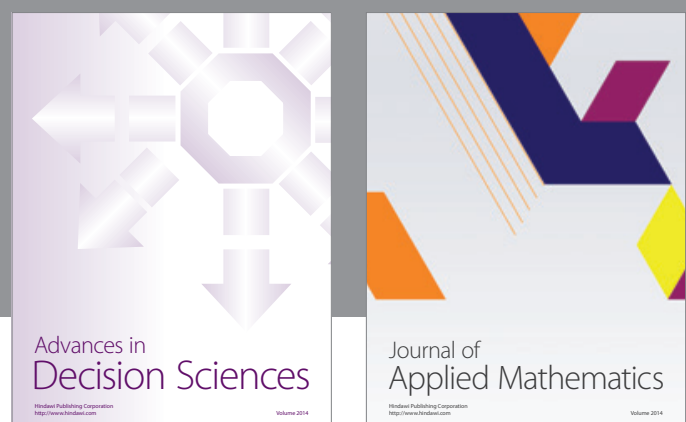

Journal of

Applied Mathematics
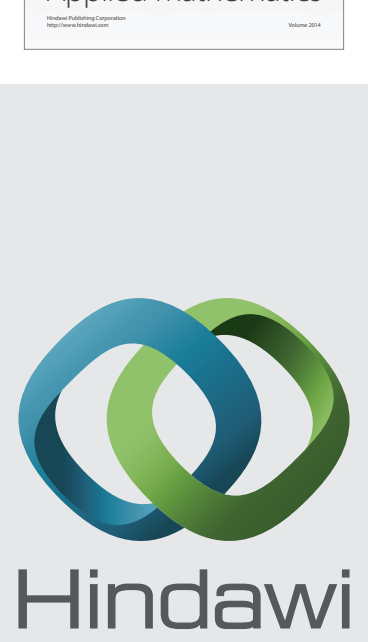

Submit your manuscripts at http://www.hindawi.com
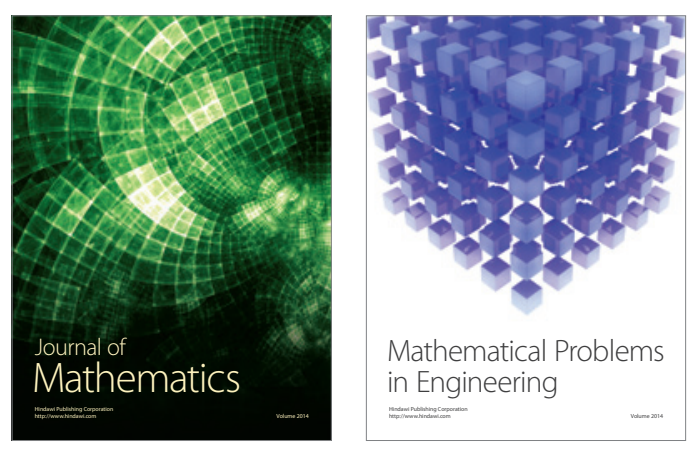

Mathematical Problems in Engineering
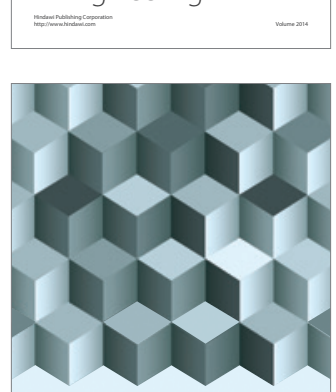

Journal of

Function Spaces
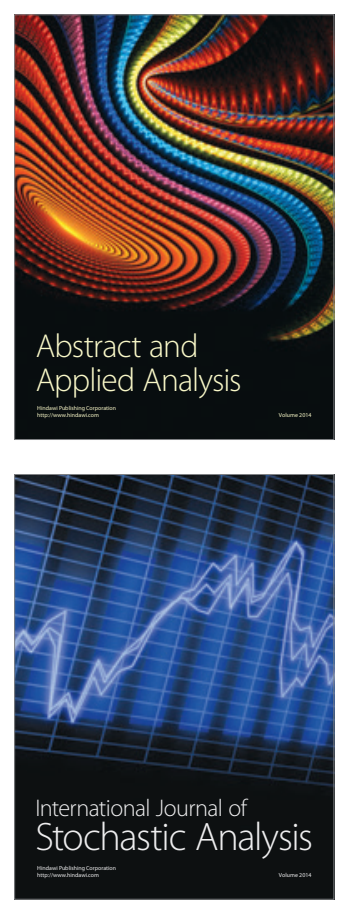

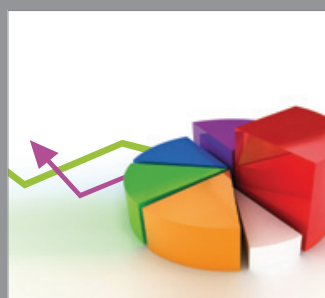

ournal of

Probability and Statistics

Promensencen
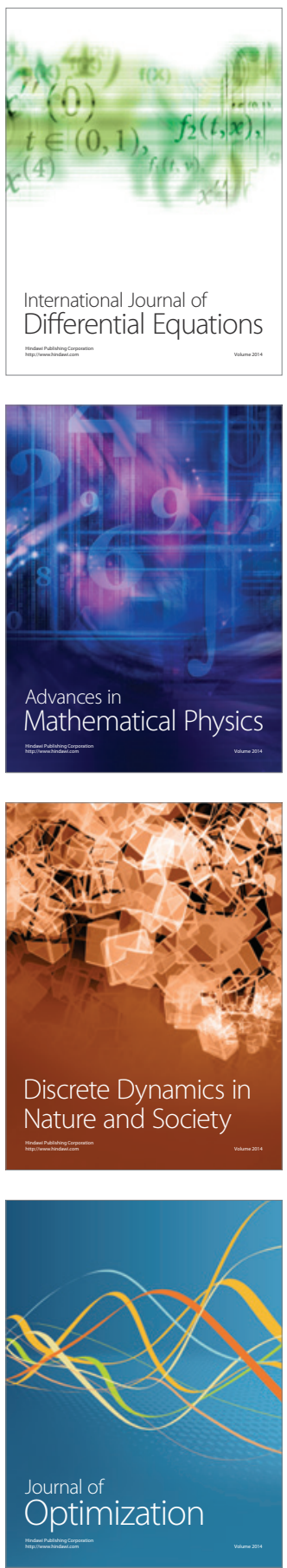